Miljenko Crnjac, University of Osijek, Osijek, Croatia

Boris Guljaš, University of Zagreb, Zagreb, Croatia

Harry I. Miller, University of Sarajevo, Sarajevo, Bosnia

current address: University of Tennessee, Chattanooga, TN 37403,

e-mail: hmiller@@utcvm.utc.edu

\title{
A POSITIVE ANSWER TO A QUESTION ABOUT THE CANTOR SET
}

\author{
Abstract \\ It is proved that $g(C \times C)$ contains an interval if $g: \mathcal{R} \times \mathcal{R} \rightarrow \mathcal{R}$ \\ satisfies appropriate conditions and $C$ is the Cantor set.
}

\section{Introduction}

Classical results of $\mathrm{H}$. Steinhaus and S. Piccard show that $A+B=\{a+b$ : $a \in A, b \in B\}$ contains an interval if either:

(a) $A$ and $B$ are both measurable subsets of the real line, each having positive Lebesgue measure, or

(b) $A$ and $B$ are both subsets of the real line possessing the Baire property, each being of the second category.

Proofs of these results can be found in Oxtoby [8, p. 21].

Many authors have generalized the above results by considering a general function $g: \mathcal{R} \times \mathcal{R} \rightarrow \mathcal{R}$ ( $\mathcal{R}$ the real line), satisfying appropriate conditions, in place of + (i.e. $g(x, y)=x+y$ ). See [1], [3] and [4] for extensions of the result of Steinhaus and [2] and [5] for extensions of the result of Piccard.

Conditions (a) and (b) are sufficient for $A+B$ to contain an interval, however neither is necessary. The classical Cantor set $C$ (see Oxtoby [8]), is of the first Baire category and has Lebesgue measure zero, but $C+C=[0,2]$. Many proofs of this fact are known. For our purposes the geometric proof of Utz [9] is most instructive.

Key Words: Cantor Set, Steinhaus and Piccard Theorems

Mathematical Reviews subject classification: Primary: 28A05

Received by the editors January 17, 1996 
Several years ago the third author posed the problem of finding sufficient conditions on $g$ so that $g(C \times C)$ must contain an interval. In this paper we give such conditions and offer two proofs, one we describe as analytic and the other as geometric.

\section{Results}

The first proof of our main result is based on the following three lemmas. Before stating Lemma 1, notice that each natural number $k, k=1,2, \ldots, 2^{n-1}$, can be written uniquely in the form: $k=1+\sum_{i=1}^{n-1} 2^{n-1-i} d_{i k}$, where $d_{i k} \in$ $\{0,1\}$. For each $k, k=1,2, \ldots, 2^{n-1}$, define $a_{k} \in C$, by the formula:

$$
a_{k}=\sum_{i=1}^{n-1} 2 d_{i k} 3^{-i}
$$

Lemma 1. The numbers $\left(a_{k}\right)_{k=1}^{2^{n-1}}$, defined as above, have the following properties:

(i) $0=a_{1}<a_{2}<\ldots<a_{2^{n-1}}<1$,

(ii) $a_{2 k}=a_{2 k-1}+2 \cdot 3^{-n+1}$ for $k=1,2, \ldots, 2^{n-2}$,

(iii) $a_{2 k-1}=3 a_{k}$ for $k=1,2, \ldots, 2^{n-2}$,

(iv) $a_{k}+\frac{2}{3}=a_{2^{n-2}+k}$ for $k=1,2, \ldots, 2^{n-2}$,

(v) $1-a_{k}=a_{2^{n-1}+1-k}+3^{-n+1}$ for $k=1,2, \ldots, 2^{n-1}$.

Proof. By (1) $a_{k}=2 \cdot 3^{-n+1} \sum_{i=1}^{n-1} 3^{n-1-i} d_{i k}$ and therefore

$$
1 \leq k=1+\sum_{i=1}^{n-1} 2^{n-1-i} d_{i k}<k^{\prime}=1+\sum_{i=1}^{n-1} 2^{n-1-i} d_{i k^{\prime}} \leq 2^{n-1}
$$

implies

$$
\sum_{i=1}^{n-1} 3^{n-1-i} d_{i k}<\sum_{i=1}^{n-1} 3^{n-1-i} d_{i k^{\prime}}
$$

which in turn implies that $a_{k}<a_{k^{\prime}}$, or that (i) is true.

Clearly, for each $k=1,2, \ldots, 2^{n-2}, d_{1 k}=0$ and therefore:

$$
k=1+\sum_{i=2}^{n-1} 2^{n-1-i} d_{i k} \quad \text { for } \quad \text { each } \quad k=1,2, \ldots, 2^{n-2}
$$


which implies

$$
2 k=2+\sum_{i=2}^{n-1} 2^{n-i} d_{i k}=2+\sum_{i=1}^{n-2} 2^{n-1-i} d_{i+1, k}=1+\sum_{i=1}^{n-1} 2^{n-1-i} d_{i k} .
$$

This implies that:

$$
d_{i, 2 k}=d_{i+1, k} \quad \text { for } \quad i=1,2, \ldots, n-2 \quad \text { and } \quad d_{n-1,2 k}=1 .
$$

In addition we have:

$$
2 k-1=1+\sum_{i=1}^{n-2} 2^{n-1-i} d_{i+1, k}=1+\sum_{i=1}^{n-1} 2^{n-1-i} d_{i, 2 k-1} .
$$

Therefore we obtain:

$$
d_{i, 2 k-1}=d_{i+1, k} \quad \text { for } \quad i=1,2, \ldots, n-2 \quad \text { and } \quad d_{n-1,2 k-1}=0 .
$$

Clearly (ii) follows immediately from (3) and (4).

From (2) and (4) it follows that for each $k=1,2, \ldots, 2^{n-2}$ we have:

$$
3 a_{k}=\sum_{i=2}^{n-1} 2 d_{i k} 3^{-i+1}=\sum_{i=1}^{n-2} 2 d_{i+1, k} 3^{-i}=\sum_{i=1}^{n-1} 2 d_{i, 2 k-1} 3^{-i}=a_{2 k-1},
$$

which is (iii).

For $1 \leq k \leq 2^{n-2}$, (1) implies that:

$$
d_{1,2^{n-2}+k}=1 \quad \text { and } \quad d_{i, 2^{n-2}+k}=d_{i k} \quad \text { if } \quad i=2,3, \ldots, n-1
$$

which yields (iv).

Since $2^{n-1}+1-k=1+\sum_{i=1}^{n-1} 2^{n-1-i}\left(1-d_{i k}\right)$ it follows that:

$$
d_{i, 2^{n-1}+1-k}=1-d_{i k} \quad \text { for } \quad i=1,2, \ldots, n-1
$$

and therefore

$$
a_{2^{n-1}+1-k}=\sum_{i=1}^{n-1} 2\left(1-d_{i k}\right) 3^{-i}=\frac{2}{3} \sum_{i=0}^{n-2} 3^{-i}-a_{k}=1-3^{-n+1}-a_{k},
$$

or (v) holds. This completes the proof of Lemma 1.

The next two lemmas contain the main ideas of our analytic proof that $g(C \times C)$ contains an interval, provided $g$ satisfies appropriate conditions.

In the following $C^{1}\left([0,1]^{2}\right)$ will denote the collection of all real valued functions $g$ defined on $[0,1]^{2}=[0,1] \times[0,1]$ whose partial derivatives, $D_{1} g$ and $D_{2} g$, exist and are continuous on $[0,1]^{2}$. 
Lemma 2. Suppose that $g \in C^{1}\left([0,1]^{2}\right)$ and $D_{2} g(x, y)>0$ for every $x, y \in$ $[0,1]$. If there exists an $n \in \mathbf{N}$ (the set of natural numbers) such that

$$
\frac{1}{3^{n}} \leq-\frac{D_{1} g(x, y)}{D_{2} g(x, y)} \leq \frac{1}{3^{n-1}} \quad \text { for } \quad \text { every } \quad x, y \in[0,1]
$$

then

$$
\left[g\left(1, a_{k}\right), g\left(0, a_{k}+3^{-n+1}\right)\right]=\bigcup_{\substack{j=2 i-1,2 i \\ m=2 e+1,2 e+2}}\left[g_{m}\left(1, a_{j}\right), g_{m}\left(0, a_{j}+3^{-n+1}\right)\right]
$$

holds for each $k=e 2^{n-1}+i, e \in\{0,1\}, \quad 1 \leq i \leq 2^{n-2}$, where $a_{k} \in C$, $k=1,2, \ldots, 2^{n-1}$ is defined as in (1) and the functions $g_{m} \in C^{1}\left([0,1]^{2}\right)$, $m=1,2,3,4$ are defined by

$$
\begin{aligned}
& g_{2 e+1}(x, y)=g\left(\frac{x+2}{3}, \frac{y+2 e}{3}\right), \\
& g_{2 e+2}(x, y)=g\left(\frac{x}{3}, \frac{y+2 e}{3}\right), \quad e=0,1 .
\end{aligned}
$$

Proof. First of all, by our hypothesis, it is clear that all the intervals in formula (7) are non trivial. Formula (7) will be obvious if we can prove the following four relations:

$$
\begin{gathered}
g\left(1, a_{k}\right)=g_{2 e+1}\left(1, a_{2 i-1}\right), g\left(0, a_{k}+3^{-n+1}\right)=g_{2 e+2}\left(0, a_{2 i}+3^{-n+1}\right), \\
g_{2 e+2}\left(1, a_{2 i-1}\right) \leq g_{2 e+1}\left(0, a_{2 i-1}+3^{-n+1}\right), \\
g_{2 e+1}\left(1, a_{2 i}\right) \leq g_{2 e+2}\left(0, a_{2 i-1}+3^{-n+1}\right), \\
g_{2 e+2}\left(1, a_{2 i}\right) \leq g_{2 e+1}\left(0, a_{2 i}+3^{-n+1}\right),
\end{gathered}
$$

where $k=e 2^{n-2}+i, e \in\{0,1\}$ and $1 \leq i \leq 2^{n-2}$.

From (8), using Lemma 1 (parts (iii) and (iv)) we have:

$$
g_{2 e+1}\left(1, a_{2 i-1}\right)=g\left(1, \frac{a_{2 i-1}}{3}+\frac{2 e}{3}\right)=g\left(1, a_{i}+\frac{2 e}{3}\right)=g\left(1, a_{k}\right) .
$$

Similarly, from (8), using Lemma 1 (parts (ii), (iii) and (iv)) we have:

$$
\begin{aligned}
g_{2 e+2}\left(0, a_{2 i}+\frac{1}{3^{n-1}}\right) & =g\left(0, \frac{a_{2 i}}{3}+\frac{2 e}{3}+\frac{1}{3^{n}}\right) \\
& =g\left(0, a_{i}+\frac{2 e}{3}+\frac{1}{3^{n-1}}\right)=g\left(0, a_{k}+\frac{1}{3^{n-1}}\right)
\end{aligned}
$$


and therefore (9) is true.

There exist $\bar{x} \in\left(\frac{1}{3}, \frac{2}{3}\right)$ and $\bar{y} \in\left(a_{k}, a_{k}+3^{-n}\right)$ such that

$$
\begin{aligned}
g_{2 e+2}\left(1, a_{2 i-1}\right) & -g_{2 e+1}\left(0, a_{2 i-1}+\frac{1}{3^{n-1}}\right) \\
& =g\left(\frac{1}{3}, \frac{a_{2 i-1}}{3}+\frac{2 e}{3}\right)-g\left(\frac{2}{3}, \frac{a_{2 i-1}}{3}+\frac{1}{3^{n}}\right) \\
& =g\left(\frac{1}{3}, a_{k}\right)-g\left(\frac{2}{3}, a_{k}+\frac{1}{3^{n}}\right) \\
& =\frac{D_{2} g(\bar{x}, \bar{y})}{3}\left(\frac{-D_{1} g(\bar{x}, \bar{y})}{D_{2} g(\bar{x}, \bar{y})}-\frac{1}{3^{n-1}}\right) \\
& \leq 0,
\end{aligned}
$$

or (10) is true.

Also there exists $\bar{x} \in(0,1)$ and $\bar{y} \in\left(a_{k}+3^{-n}, a_{k}+23^{-n}\right)$ such that

$$
\begin{aligned}
g_{2 e+1}\left(1, a_{2 i}\right) & -g_{2 e+2}\left(0, a_{2 i-1}+\frac{1}{3^{n-1}}\right) \\
& =g\left(1, \frac{a_{2 i}}{3}+\frac{2 e}{3}\right)-g\left(0, \frac{a_{2 i-1}}{3}+\frac{2 e}{3}+\frac{1}{3^{n}}\right) \\
& =g\left(1, a_{k}+\frac{2}{3^{n}}\right)-g\left(0, a_{k}+\frac{1}{3^{n}}\right) \\
& =D_{2} g(\bar{x}, \bar{y})\left(\frac{1}{3^{n}}+\frac{D_{1} g(\bar{x}, \bar{y})}{D_{2} g(\bar{x}, \bar{y})}\right) \\
& \leq 0,
\end{aligned}
$$

or (11) is true.

In a similar way, there exists $\bar{x} \in\left(\frac{1}{3}, \frac{2}{3}\right)$ and $\bar{y} \in\left(a_{k}+\frac{2}{3^{n}}, a_{k}+\frac{1}{3^{n-1}}\right)$ such that

$$
\begin{aligned}
g_{2 e+2}\left(0, a_{2 i}\right) & -g_{2 e+1}\left(0, a_{2 i}+\frac{1}{3^{n-1}}\right) \\
& =g\left(\frac{1}{3}, \frac{a_{2 i}}{3}+\frac{2 e}{3}\right)-g\left(\frac{2}{3}, \frac{a_{2 i}}{3}+\frac{2 e}{3}+\frac{1}{3^{n}}\right) \\
& =g\left(\frac{1}{3}, a_{k}+\frac{2}{3^{n}}\right)-g\left(\frac{2}{3}, a_{k}+\frac{1}{3^{n-1}}\right) \\
& =\frac{D_{2} g(\bar{x}, \bar{y})}{3}\left(\frac{-D_{1} g(\bar{x}, \bar{y})}{D_{2} g(\bar{x}, \bar{y})}-\frac{1}{3^{n-1}}\right) \\
& \leq 0
\end{aligned}
$$


or (12) holds, completing the proof of Lemma 2.

Our next lemma gives an exact description of the set $g(C, C)$.

Lemma 3. Suppose that $g \in C^{1}\left([0,1]^{2}\right)$ and that $D_{2} g(x, y)>0$ for every $x, y \in[0,1]$. For each $n \in \mathbf{N}$ the following statements are true, where the numbers $a_{k}\left(k=1,2, \ldots, 2^{n-1}\right)$ are defined by formula (1):

(i) If $\frac{1}{3^{n}} \leq \frac{-D_{1} g(x, y)}{D_{2} g(x, y)} \leq \frac{1}{3^{n-1}}$ for every $x, y \in[0,1]$, then

$$
g(C, C)=\bigcup_{k=1}^{2^{n-1}}\left[g\left(1, a_{k}\right), g\left(0, a_{k}+\frac{1}{3^{n-1}}\right)\right] .
$$

(ii) If $3^{n-1} \leq \frac{-D_{1} g(x, y)}{D_{2} g(x, y)} \leq 3^{n}$ for every $x, y \in[0,1]$, then

$$
g(C, C)=\bigcup_{k=1}^{2^{n-1}}\left[g\left(a_{k}+\frac{1}{3^{n-1}}, 0\right), g\left(a_{k}, 1\right)\right] .
$$

(iii) If $\frac{-1}{3^{n-1}} \leq \frac{-D_{1} g(x, y)}{D_{2} g(x, y)} \leq \frac{-1}{3^{n}}$ for every $x, y \in[0,1]$, then

$$
g(C, C)=\bigcup_{k=1}^{2^{n-1}}\left[g\left(0, a_{k}\right), g\left(1, a_{k}+\frac{1}{3^{n-1}}\right)\right] .
$$

(iv) If $-3^{n} \leq \frac{-D_{1} g(x, y)}{D_{2} g(x, y)} \leq-3^{n-1}$ for every $x, y \in[0,1]$, then

$$
g(C, C)=\bigcup_{k=1}^{2^{n-1}}\left[g\left(a_{k}, 0\right), g\left(a_{k}+\frac{1}{3^{n-1}}, 1\right)\right] .
$$

Proof. First we will show that (i) holds, and then the remaining statements will follow at once.

Suppose that $x_{0}, y_{0} \in C$. then there exists a natural number $k, k=$ $1,2, \ldots, 2^{n-1}$, such that $a_{k} \leq y_{0} \leq a_{k}+3^{-n+1}$.

Since $D_{2} g(x, y)>0$ for all $x, y \in[0,1]$ we have

$$
g\left(x_{0}, a_{k}\right) \leq g\left(x_{0}, y_{0}\right) \leq g\left(x_{0}, a_{k}+3^{-n+1}\right) .
$$

Furthermore (i) implies that $D_{1} g(x, y)<0$ for all $x, y \in[0,1]$ and since $x_{0} \in$ $[0,1]$ we have

$$
g\left(1, a_{k}\right) \leq g\left(x_{0}, a_{k}\right) \quad \text { and } \quad g\left(x_{0}, a_{k}+3^{-n+1}\right) \leq g\left(0, a_{k}+3^{-n+1}\right) .
$$


From (13) and (14) it follows that $g\left(1, a_{k}\right) \leq g\left(x_{0}, y_{0}\right) \leq g\left(0, a_{k}+3^{-n+1}\right)$ and therefore

$$
g(C, C) \subset \bigcup_{k=1}^{2^{n-1}}\left[g\left(1, a_{k}\right), g\left(0, a_{k}+\frac{1}{3^{n-1}}\right)\right] .
$$

We will now show that the reverse inclusion holds.

For any sequence $\left\langle j_{i}: i \in \mathbf{N}\right\rangle$ with $j_{i} \in\{1,2,3,4\}$ we will define inductively a sequence of functions from $C^{1}\left([0,1]^{2}\right)$ in the following manner. The function $g_{j_{i}}$ is defined as in formula (8) in the statement of Lemma 2 , and for $p \geq 2$ by

$$
g_{j_{1}, j_{2}, \ldots, j_{p}}=\left\langle g_{j_{1}, j_{2}, \ldots, j_{p-1}}\right\rangle_{j_{p}} .
$$

By induction it is easy to see that

$$
g_{j_{1}, j_{2}, \ldots, j_{p}}(x, y)=g\left(\sum_{i=1}^{p} \frac{2 d_{i}}{3^{i}}+\frac{x}{3^{p}}, \sum_{i=1}^{p} \frac{2 b_{i}}{3^{i}}+\frac{y}{3^{p}}\right)
$$

where

$$
\begin{array}{llll}
d_{i}=1, & b_{i}=0 & \text { if } & j_{i}=1 \\
d_{i}=0, & b_{i}=0 & \text { if } & j_{i}=2 \\
d_{i}=1, & b_{i}=1 & \text { if } & j_{i}=3 \\
d_{i}=0, & b_{i}=1 & \text { if } & j_{i}=4 .
\end{array}
$$

Clearly by the Mean Value Theorem we have:

$$
\begin{gathered}
\left|g_{j_{1}, j_{2}, \ldots, j_{p}}\left(1, a_{k_{p}}\right)-g_{j_{1}, j_{2}, \ldots, j_{p}}\left(0, a_{k_{p}}+\frac{1}{3^{n-1}}\right)\right| \\
\leq M_{1} \frac{1}{3^{p}}+M_{2} \frac{1}{3^{p+n-1}}
\end{gathered}
$$

for every $p \in \mathbf{N}$, where $M_{i}=\max _{x, y \in[0,1]}\left|D_{i} g(x, y)\right|, \quad i=1,2$.

For each $r \in\left[g\left(1, a_{k}\right), g\left(0, a_{k}+3^{-n+1}\right)\right]$, it follows from formula (7) in Lemma 2 that there exists a sequence $\left\langle j_{i}: i \in \mathbf{N}\right\rangle$ with the property that

$$
r \in\left[g_{j_{1}, j_{2}, \ldots, j_{p}}\left(1, a_{k_{p}}\right), g_{j_{1}, j_{2}, \ldots, j_{p}}\left(0, a_{k_{p}}+\frac{1}{3^{n-1}}\right)\right]
$$

for all $p \in \mathbf{N}$. From (19), using (18) we have

$$
r=\lim _{p \rightarrow \infty} g_{j_{1}, j_{2}, \ldots, j_{p}}\left(1, a_{k_{p}}\right) .
$$


By formula (16) and the continuity of $g$, this in turn implies that

$$
\begin{aligned}
r & =\lim _{p \rightarrow \infty} g\left(\sum_{i=1}^{p} \frac{2 d_{i}}{3^{i}}+\frac{1}{3^{p}}, \sum_{i=1}^{p} \frac{2 b_{i}}{3^{i}}+\frac{a_{k_{p}}}{3^{p}}\right) \\
& =g\left(\sum_{i=1}^{\infty} \frac{2 d_{i}}{3^{i}}, \sum_{i=1}^{\infty} \frac{2 b_{i}}{3^{i}}\right)=g\left(x_{0}, y_{0}\right),
\end{aligned}
$$

where

$$
x_{0}=\sum_{i=1}^{\infty} \frac{2 d_{i}}{3^{i}} \quad \text { and } \quad y_{0}=\sum_{i=1}^{\infty} \frac{2 b_{i}}{3^{i}}
$$

are both in $C$. Therefore the proof of (i) is complete.

Clearly $1-C=C$. Using this fact, (ii), (iii) and (iv) can be shown by using (i) with $g(1-y, 1-x), \quad g(1-x, y)$ and $g(y, 1-x)$, respectively, playing the role of $g(x, y)$. This completes the proof of Lemma 3.

We are now in a position to give an easy analytic proof of the fact that $g(C, C)$ contains an interval if $g$ satisfies appropriate conditions. We then provide a second geometric proof.

Theorem 4. Suppose $g \in C^{1}\left([0,1]^{2}\right)$. If there exist $x_{0}, y_{0} \in C$ such that $D_{1} g\left(x_{0}, y_{0}\right) \neq 0, D_{2} g\left(x_{0}, y_{0}\right) \neq 0$ and one of the following conditions holds:

(i) $\left|D_{1} g\left(x_{0}, y_{0}\right) / D_{2} g\left(x_{0}, y_{0}\right)\right| \neq 3^{n}$ for each $n \in \mathbf{Z}$,

(ii) $\left(x_{0}, y_{0}\right)$ is the location of a local extremum of the function $D_{1} g / D_{2} g$, then $g(C, C)$ contains an interval.

First Proof (Analytic). Our first proof uses Lemmas 1, 2 and 3. Without loss of generality we can assume that $D_{2} g\left(x_{0}, y_{0}\right)>0$. If either condition (i) or condition (ii) holds then there exists a neighborhood $U\left(x_{0}, y_{0}\right)$ of $\left(x_{0}, y_{0}\right)$ and an $n \in \mathbf{N}$ such that,

$$
\begin{aligned}
D_{2} g(x, y) & >0 \text { for all }(x, y) \in U\left(x_{0}, y_{0}\right) \\
\frac{-D_{1} g(x, y)}{D_{2} g(x, y)} & \in I_{n},
\end{aligned}
$$

where $I_{n}$ is one of the following four intervals: $\left[1 / 3^{n}, 1 / 3^{n-1}\right],\left[3^{n-1}, 3^{n}\right]$, $\left[-1 / 3^{n-1},-1 / 3^{n}\right]$ and $\left[-3^{n},-3^{n-1}\right]$. As we saw in the proof of Lemma 3 , since $x_{0}, y_{0} \in C$, there exists $m \in \mathbf{N}$ and $k_{1}, k_{2} \in\left\{1,2, \ldots, 2^{m-1}\right\}$ such that

$$
\left(x_{0}, y_{0}\right) \in\left[a_{k_{1}}, a_{k_{1}}+\frac{1}{3^{m-1}}\right] \times\left[a_{k_{2}}, a_{k_{2}}+\frac{1}{3^{m-1}}\right] \subset U\left(x_{0}, y_{0}\right) .
$$


The proof of the theorem is completed by applying Lemma 3 with the function $g\left(a_{k_{1}}+x / 3^{m-1}, a_{k_{2}}+y / 3^{m-1}\right)$ playing the role of $g(x, y)$. We remark that $x, y \in C$ imply that (by (v) from Lemma 1) $a_{k_{1}}+x / 3^{m-1}, a_{k_{2}}+y / 3^{m-1}$ $\in C$.

The following lemma, based on a result of Utz [9], will be needed in the second (geometric) proof of our theorem.

Lemma 5. If $f \in C^{1}([0,1])$ and either (i) $1 \leq\left|f^{\prime}(x)\right| \leq 3$ for all real numbers $x$ or (ii) $1 / 3 \leq\left|f^{\prime}(x)\right| \leq 1$ for all real numbers $x$, is satisfied then $F=\{(x, f(x)): x \in \mathcal{R}\}$, the graph of $f$, satisfies the condition $F \cap(C \times C) \neq \emptyset$ provided $F \cap([0,1] \times[0,1]) \neq \emptyset$.

Proof. Since $F \cap([0,1] \times[0,1]) \neq \emptyset$ and either (i) or (ii) holds $F \cap S_{1 i} \neq \emptyset$ for some $i \in\{1,2,3,4\}$, say $F \cap S_{1 i_{1}} \neq \emptyset$, where $\left\{S_{1 i}: i=1,2,3,4\right\}$ are the four "corner" squares (clockwise) of $[0,1] \times[0,1]$ formed in the first step of the construction of $C \times C$.

Sketch of $[0,1] \times[0,1]$ :

\begin{tabular}{|l|l|l|}
\hline$S_{11}$ & & $S_{12}$ \\
\hline & & \\
\hline$S_{14}$ & & $S_{13}$ \\
\hline
\end{tabular}

$\begin{array}{llll}0 & 1 / 3 & 2 / 3 & 1\end{array}$

Since $F \cap S_{1 i_{1}} \neq \emptyset$ and either (i) or (ii) holds, it follows that $F \cap S_{2 i} \neq \emptyset$ for some $i \in\{1,2,3,4\}$, say $F \cap S_{2 i_{2}} \neq \emptyset$ where $\left\{S_{2 i}: \quad i=1,2,3,4\right\}$ are the four "corner" squares (clockwise) of $S_{1 i_{1}}$ formed in the second step of the construction of $C \times C$.

Sketch of $S_{1 i_{1}}$ :

\begin{tabular}{|l|l|l|}
\hline$S_{21}$ & & $S_{22}$ \\
\hline & & \\
\hline$S_{24}$ & & $S_{23}$ \\
\hline
\end{tabular}

Continue this process. Then we obtain a sequence of nested squares $\left\{S_{n i_{n}}\right.$ : $n \in \mathbf{N}\}$ satisfying: $F \cap S_{n i_{n}} \neq \emptyset$ for each $n \in \mathbf{N}$. Furthermore clearly, $\cap_{n=1}^{\infty} S_{n i_{n}}=\left\{\left(x_{0}, y_{0}\right)\right\}, x_{0}, y_{0} \in C$ and $\left(x_{0}, y_{0}\right) \in F$. Therefore $F \cap(C \times C) \neq \emptyset$, completing the proof of Lemma 4.

SECOND Proof (GEOMETRIC). If either condition (i) or (ii), in the statement of our theorem holds then there exists a neighborhood $U\left(x_{0}, y_{0}\right)$ of $\left(x_{0}, y_{0}\right)$ and an $n \in \mathbf{N}$ such that

$$
\left|D_{1} g(x, y) / D_{2} g(x, y)\right| \in I_{n} \quad \text { for } \quad \text { all } \quad\left(x_{0}, y_{0}\right) \in U\left(x_{0}, y_{0}\right)
$$


where $I_{n}$ is an interval of the form $\left[1 / 3^{n}, 1 / 3^{n-1}\right]$ or $\left[3^{n-1}, 3^{n}\right]$. Notice, that without loss of generality we may assume, in addition to $x_{0}, y_{0} \in C$, that $x_{0}, y_{0}$ satisfy: $0<x_{0}, y_{0}<1$ and $U\left(x_{0}, y_{0}\right) \subset(0,1) \times(0,1)$. From this it follows that there exists $c_{1}, c_{2} \in C$ and $m \in \mathbf{N}$ such that:

$$
T_{m}=\left[c_{1}, c_{2}+\frac{1}{3^{m}}\right] \times\left[c_{1}, c_{2}+\frac{1}{3^{m}}\right]
$$

is one of the $4^{m}$ squares in the $m$-th stage of the construction of $C \times C$ and $T_{m} \subset U\left(x_{0}, y_{0}\right)$.

Now let $h$ denote the translate of $g$ to $S_{m}:=\left[0,1 / 3^{m}\right] \times\left[0,1 / 3^{m}\right]$, i.e. $h(x, y)=g\left(c_{1}+x, c_{2}+y\right)$ for each $(x, y) \in S_{m}$. Notice that $c_{1}+x, c_{2}+y \in C$ whenever $x, y \in C \cap\left[0,1 / 3^{m}\right]$.

Next, suppose $0<a, b<1, a$ and $b$ fixed and consider $F(x, y)=h(a x, b y)$ for each $(x, y) \in S_{m}$. Denote $g\left(c_{1}, c_{2}\right)$ by $t_{0}$. There exists $\delta, \varepsilon>0$, with $\delta<1 / 3^{m}$, such that, for each $t \in I$ (where $I$ is either the interval $\left(t_{0}, t_{0}+\varepsilon\right]$ or the interval $\left.\left[t_{0}-\varepsilon\right), t_{0}\right]$, depending on the signs of $D_{1} g\left(x_{0}, y_{0}\right)$ and $\left.D_{2} g\left(x_{0}, y_{0}\right)\right)$ and $x \in[0, \delta)$ there exists a unique $y_{t}(x) \in\left[0,1 / 3^{m}\right]$ such that

$$
F\left(x, y_{t}(x)\right)=t
$$

Therefore, for each fixed $t \in I$

$$
h\left(a x, b y_{t}(x)\right)=t \quad \text { for } \quad \text { all } \quad x \in[0, \delta)
$$

This implies that

$$
y_{t}^{\prime}(x)=-\frac{a D_{1} h\left(a x, b y_{t}(x)\right)}{b D_{2} h\left(a x, b y_{t}(x)\right)} \quad \text { for } \quad \text { all } \quad x \in[0, \delta)
$$

Let $E$ denote the number $\left|D_{1} g\left(x_{0}, y_{0}\right) / D_{2} g\left(x_{0}, y_{0}\right)\right|$. We now consider three cases using formula (24).

1a. If $3^{n-1} \leq E \leq 3^{n}$ and $n=0$, set $a=1$ and $b=1$, then by (24) we have $1 / 3 \leq\left|y_{t}^{\prime}(x)\right| \leq 1$ for every $x \in[0, \delta)$ and every $t \in I$.

1b. If $3^{n-1} \leq E \leq 3^{n}$ and $n=1$, set $a=1$ and $b=1$, then by (24) we have $1 \leq\left|y_{t}^{\prime}(\bar{x})\right| \leq 3$ for every $x \in[0, \delta)$ and every $t \in I$.

2. If $3^{n-1} \leq E \leq 3^{n}$ and $n>1$, set $a=3^{-n}$ and $b=1$, then by (24) we have $1 / 3 \leq\left|y_{t}^{\prime}(x)\right| \leq 1$ for every $x \in[0, \delta)$ and every $t \in I$.

3. If $3^{n-1} \leq E \leq 3^{n}$ and $n<0$, set $a=1$ and $b=3^{n}$, then by (24) we have $1 / 3 \leq\left|\overline{y_{t}^{\prime}}(x)\right| \leq 1$ for every $x \in[0, \delta)$ and every $t \in I$. 
Now, for each $t \in I$, we will examine $G_{t}$, the graph of $y_{t}$. Taking $\delta=$ $1 / 3^{n_{0}}, n_{0}>m$ and $\varepsilon$ sufficiently small, $G_{t}$ (for each $t \in I$ ) "hits" $\left[0,1 / 3^{n_{0}}\right] \times$ $\left[0,1 / 3^{n_{0}}\right]$, that is

$$
G_{t} \cap\left[0, \frac{1}{3^{n_{0}}}\right] \times\left[0, \frac{1}{3^{n_{0}}}\right] \neq \emptyset \quad \text { for } \quad \text { every } \quad t \in I .
$$

Then, by the argument used in the proof of Lemma 4 , for each $t \in I$, there exists $\left(\bar{x}_{t}, \bar{y}_{t}\right) \in C \times C$ such that $y_{t}\left(\bar{x}_{t}\right)=\bar{y}_{t}$ and therefore

$$
h\left(a \bar{x}_{t}, b \bar{y}_{t}\right)=t \quad \text { for } \quad \text { every } \quad t \in I .
$$

But $h\left(a \bar{x}_{t}, b \bar{y}_{t}\right)=g\left(c_{1}+a \bar{x}_{t}, c_{2}+b \bar{y}_{t}\right)$. By the definition of $c_{1}$ and $c_{2}$, the fact that $\bar{x}_{t}, \bar{y}_{t} \in C \cap\left[0,1 / 3^{n_{0}}\right]$ and $a, b \in\left\{1,1 / 3,1 / 3^{2}, \ldots\right\}$ in all cases, it follows that

$$
c_{1}+a \bar{x}_{t}, c_{2}+b \bar{y}_{t} \in C
$$

and therefore $g(C \times C) \supseteq I$, completing our second proof.

We conclude this paper with the following observations.

Remark 1. First of all we will give an example of our theorem. Let $g(x, y)=$ $x y, x_{0}=2 / 3$ and $y_{0}=1 / 3$. Clearly condition (i) of our theorem holds and therefore $g(C, C)$ contains an interval.

Remark 2. For each $n \in \mathbf{N}$, let $C_{2 n+1}$ denote the "Cantor-like" subset of $[0,1]$ obtained by removing the middle $\frac{1}{2 n+1}$-th of each interval at each step of the "Cantor-like" construction. In particular $C_{3}=C$ (the ordinary Cantor set) and $C_{2 n+1}$ has Lebesgue measure zero for $n \in \mathbf{N}$. We remark that a result like our Theorem can be proved for each set $C_{2 n+1}, n \in \mathbf{N}$.

Remark 3. There exists a perfect set $A, A \subset[0,1]$, and a Baire measure $\mu$ (i.e., a measure induced by a nondecreasing continuous function), such that $\mu(A)>0$ and such that $A-A$ has Lebesgue measure zero (and therefore $A-A$ contains no interval). This result can be found in [6], where it was conjectured that $g(C \times C)$ contains an interval provided that $g$ satisfies appropriate conditions.

Remark 4. A set $A, A \subset \mathcal{R}$, is called a universal null set if $\mu(A)=0$ for each Baire measure $\mu$. There exists a universal null set $A$ such that $A-A=\mathcal{R}$. This result was shown in [7]. 


\section{References}

[1] P. Erdős and J. C. Oxtoby, Partitions of the plane into sets having positive measures in every non-null product set, Trans. Amer. Math. Soc., 79 (1955), 91-102.

[2] Z. Kominek, Some generalization of the theorem of S. Piccard, Prace Nauk. Univ. Śląski Katowice, Prace Mat., 4 (1973), 31-33.

[3] M. E. Kuczma and M. Kuczma, An elementary proof and an extension of a theorem of Steinhaus, Glas. Mat. Ser. III, 6(26) (1971), 11-18.

[4] H. I. Miller, Generalization of a classical theorem of measure theory, Radovi Akad. Nauka i Umjetnosti B i H, 45 (1973), 45-48.

[5] H. I. Miller, Relationships between various gauges of the size of sets of real numbers, Glas. Mat. Ser. III, 9(29) (1974), 59-64.

[6] H. I. Miller, Relationships between various gauges of the size of sets of real numbers II, Radovi Akad. Nauka i Umjetnosti B i H, 59 (1976), 37-48.

[7] H. I. Miller, A universal null set whose Steinhaus distance set is $[0, \infty)$, Radovi Akad. Nauka i Umjetnosti B i H, 61 (1978), 37-40.

[8] J. C. Oxtoby, Measure and Category, Springer-Verlag, New York, 1970.

[9] W. R. Utz, The distance set of the Cantor discontinuum, Amer. Math. Monthly, June (1951), 407-408. 